\title{
Coherence of VCSEL's for Holographic Interconnects
}

\author{
J. L. A. Chilla, B. Benware, M. E. Watson, P. Stanko, J. J. Rocca, Member, IEEE, \\ C. Wilmsen, Member, IEEE, S. Feld, and R. Leibenguth
}

\begin{abstract}
Temporal and spatial coherence measurements were conducted on the emission from AlGaAs-GaAs vertical-cavity surface-emitting lasers (VCSEL's). Results of both CW and pulsed conditions from devices of 8- and 15- $\mu \mathrm{m}$ diameter are reported. Coherence length of $0.6 \mathrm{~mm}$ for multi-mode and $\sim 75$ $\mathrm{cm}$ for single-mode were obtained. Thermal chirp reduces the single-mode coherence to $16 \mathrm{~mm}$ for $5 \mathrm{~ns}$ pulses. For holograms smaller than $0.6 \mathrm{~mm}$ in size the density of information that can be read by the VCSEL's is diffraction-limited by the size of the holographic element.
\end{abstract}

\section{INTRODUCTION}

$\mathbf{T}$ HERE is increasing interest in free-space global holographic interconnects for optical computers [1], [2] and two-dimensional (2-D) arrays of vertical-cavity surfaceemitting lasers (VCSEL's) coupled with 2-D computergenerated hologram arrays to route the beams [3]. VCSEL's appear well suited to this application since they can be implemented in large-area 2-D emitter arrays and can be designed for single-mode emission. However, the maximum single-mode power attainable is limited by the onset of higherorder transverse mode emission [4], which can significantly degrade the coherence characteristics, when the VCSEL's are operated at high currents. In this letter we report a study of the coherence of the emission of gain-guided AlGaAs-GaAs VCSEL's with an emission wavelength around $840 \mathrm{~nm}$. We performed both temporal and spatial coherence measurements on devices of 8 and $15 \mu \mathrm{m}$ in active area diameter, for $\mathrm{CW}$ and pulsed excitation conditions. In the latter case, square electrical pulses as short as $5 \mathrm{~ns}$ were used to simulate the conditions of an optical interconnect.

The VCSEL's used in the study had an n-type mirror consisting of 32 periods of $\mathrm{AlAs}-\mathrm{AlGaAs}_{0.58}-\mathrm{AlGaAs}_{0.3}$, a five-period $\mathrm{GaAs}-\mathrm{AlGaAs}_{0.3}$ quantum well active region and 23 periods of $\mathrm{AlAs}-\mathrm{AlGaAs}_{0.58}-\mathrm{AlGaAs}_{0.3}$ p-type mirror. The top-surface emitting, gain-guided laser structures were patterned using a multiple-energy proton implant schedule. Devices with 8- and $15-\mu \mathrm{m}$-diameter active areas were masked

Manuscript received October 31, 1994; revised January 30, 1995. This work was supported by the National Science Foundation, Center for Optoelectronic Computer Systems through N.S.F. Grant no. EEC 9015128, and by the Colorado Advanced Technology Institute, UCB Ref. no. 0594.75.0738B

J. L. A. Chilla, B. Benware, M. E. Watson, P. Stanko, J. J. Rocca, C. Wilmsen, and S. Feld are with the Center for Optoelectronic Computing Systems and Department of Electrical Engineering, Colorado State University, Fort Collins, CO 80523 USA

R. Leibenguth is with AT\&T Bell Laboratories, Breiningsville, PA 18031 USA.

IEEE Log Number 9410457. using 4- $\mu \mathrm{m}$-thick electroplated gold. The devices were isolated using thick photoresist masks of 20 and $50 \mu \mathrm{m}$ diameter, respectively, for the lower-energy implants. The top p-type ohmic contact was evaporated $\mathrm{Be}-\mathrm{Au}$, with 15 - and 28$\mu \mathrm{m}$-diameter apertures, respectively. A Ge-Au-Ni-Au n-type ohmic contact was evaporated on the back of the substrate. As is well known [5], the output power of the VCSEL's decreases at high currents due to temperature-induced mismatch of the mirror reflectivity and gain spectra. For the 15- $\mu \mathrm{m}$ VCSEL the emission was multi-mode at high currents as expected for its relatively large diameter. Individual devices of a nominally 8$\mu \mathrm{m}$ VCSEL showed different behaviors due to local variations in the proton implantation defining the diameter of the active area. Some of the $8-\mu \mathrm{m}$ devices studied were single-mode throughout their entire operating range while we observed multi-mode emission in other 8- $\mu \mathrm{m}$ VCSEL's. The wavelength separation $(0.3 \mathrm{~nm}$ and $0.6 \mathrm{~nm}$ for the $15-\mu \mathrm{m}$ and $8-\mu \mathrm{m}$ VCSEL, respectively) and spatial profiles of the different modes were similar to those previously reported in other gain-guided VCSEL's [4].

Measurements of the temporal coherence of the devices were carried out utilizing a Michelson interferometer [6] and measuring the visibility of the resulting interference fringes as a function of the difference in optical path (or equivalent time delay) between both branches of the interferometer. The interference pattern was acquired with a linear array of 1024 photodiodes and normalized by taking into account the intensity profiles of both individual beams. Fig. 1(a) shows the dependence of the visibility of the fringes for the $15-\mu \mathrm{m}$ VCSEL at two different currents. At the lower current, $6 \mathrm{~mA}$, the laser operates in a single mode, and thus the visibility is observed to decay in an approximately exponential fashion, with a coherence time of $1 \mathrm{~ns}(30 \mathrm{~cm})$. When more than one mode is oscillating the results are very different, as shown in Fig. 1(a) for the 15- $\mu \mathrm{m}$ VCSEL operating at $12 \mathrm{~mA}$. In this case the visibility is modulated with a period of $7.8 \mathrm{ps}$ corresponding to the spectral separation of the modes. Here the amplitude of the modulation is set by the relative amplitudes of the modes involved, while the mean value of the visibility decays with increasing delay in a scale similar to that of the single-mode visibility. The consequence of this mode beating is that the coherence of the VCSEL's changes appreciably in a scale of less than a millimeter ( $3.3 \mathrm{ps)}$ as shown in the inset in Fig. 1(a). For the 8- $\mu \mathrm{m}$ multi-mode VCSEL the results are similar with larger mode separation and shorter period in the modulation of the visibility. 


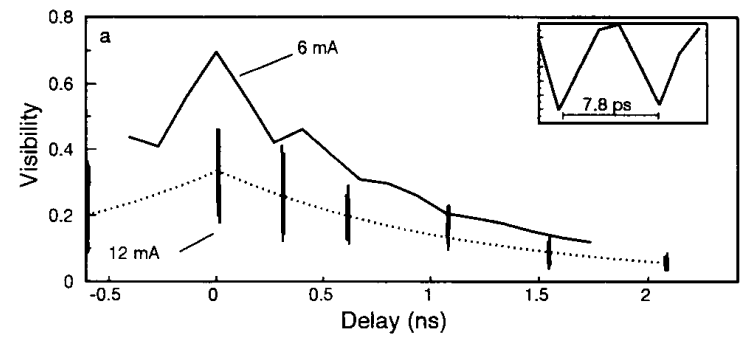

(a)

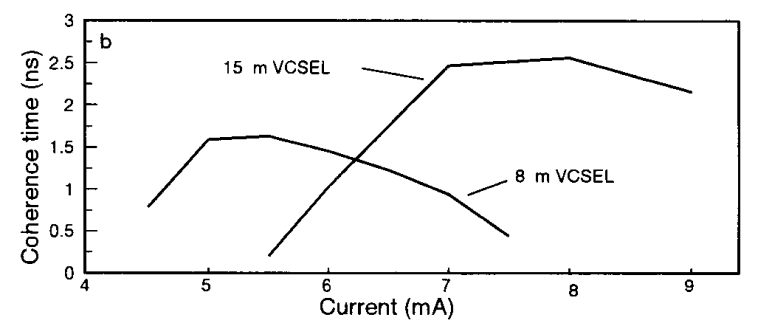

(b)

Fig. 1. (a) Visibility of the fringes as a function of the optical path difference in a Michelson interference experiment for the 15- $\mu \mathrm{m}$ VCSEL. At $6 \mathrm{~mA}$ the emission is single-mode while at $12 \mathrm{~mA}$ two modes oscillate simultaneously. The inset shows the corresponding modulation of the visibility due to mode beating. (b) Current dependence of the coherence time for the single-mode $8-\mu \mathrm{m}$ VCSEL and the single-mode portion of the operating range of the $15-\mu \mathrm{m}$ VCSEL.

For single-mode lasers, the coherence time is obtained from exponential fitting of the data. Fig. 1(b) shows the dependence of the coherence on the injected current for the single-mode $8-\mu \mathrm{m}$ VCSEL and the single-mode region of the operating range of the $15-\mu \mathrm{m}$ VCSEL. The coherence time of VCSEL's is fundamentally limited [6] to a few nanoseconds due to their short photon lifetimes ( 5 ps typical). The thermal problems limiting the single mode output power further limit the coherence, with a measured linewidth-power product of 60-110 Mhz.mW which was not constant due to thermal effects [7]. A smaller linewidth-power product was previously reported for InGaAs VCSEL's [8], [9].

We atso measured the temporal coherence at conditions similar to those in which the laser would be used in interconnects, with a sub-threshold DC bias $(2.8 \mathrm{~mA}, 90 \%$ of the threshold current) on top of which we added $2 \mathrm{~V}$ amplitude $(7 \mathrm{~mA}$ peak current) pulses of various durations. As can be seen in Fig. 2(a), the linewidth increases with pulse length at a rate of 1.96 $\mathrm{nm} / \mu$ s due to thermal chirp caused by changes in temperature during the pulse [4]. The change in temperature due to the peak current was measured to produce a change in wavelength of $\sim 5 \mathrm{~nm}$ in dc conditions, from where a thermal time constant of $\sim 2.5 \mu \mathrm{s}$ can be estimated. Assuming that the spectral broadening is only thermal in origin, and using the measured broadening rate, we calculated the expected coherence length [6]. The results, plotted in solid line in Fig. 2(b) show good agreement with the measured coherence lengths, confirming the hypothesis of thermal broadening being dominant. Minor differences are due to the fact (not taken into account) that the line shape changes with pulse length. It should be noticed that

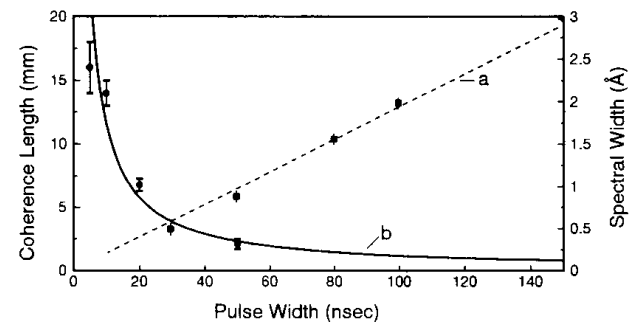

Fig. 2. (a) Measured linewidth as a function of pulse length. The region below $30 \mathrm{~ns}$ is not resolved by the spectrometer. (b) Coherence length: Measured (data points) and computed assuming thermal chirp (solid line).

our experiments were performed at a relatively low repetition rate of $80 \mathrm{kHz}$, thus giving the device time to cool down between pulses. From the thermal chirp point of view, this is a worst-case situation, as the changes in temperature during the pulse would be smaller at higher rates.

The spatial coherence of the VCSEL's was measured from Young interference experiments in which the visibility of the fringes was measured as a function of the separation of two $300-\mu$ m-diameter pinholes placed $32 \mathrm{~mm}$ apart from the laser. One pinhole was located at the center of the emission profile, while the second could be adjusted to subtend angles from 2 to 8 degrees as seen by the VCSEL, thus ensuring sampling of most of the far field emission profile of the VCSEL's studied, which had a FWHM angle between 6 and 8 degrees. As in the case of temporal coherence, a normalization procedure was used to avoid artifacts caused by the changing intensity of the interfering beams.

Fig. 3 shows the spatial coherence of the multi-mode 8and $15-\mu \mathrm{m}$ VCSEL's as a function of current. The coherence semi-angle is defined from the decay rate of the visibility as a function of subtended angle. In the case of the 8$\mu \mathrm{m}$ VCSEL, for low and high currents the output power is low, and consequently the visibility drops faster with increasing subtended angle. For intermediate currents, and even at currents as low as $10 \%$ above threshold the visibility is high and fairly independent of the subtended angle, an indication that the output is spatially coherent within a solid angle larger than the FWHM divergence angle of the beam. Similar results were obtained for all the VCSEL's studied. As shown in the figure for the $15-\mu \mathrm{m}$ VCSEL, the onset of multi-mode emission has no significant effect observed on the spatial coherence, in contrast with the dramatic effect this has on the temporal coherence. This can be explained by realizing that the relative phase between different modes is not relevant here, and thus changes in wavelength of the observed magnitudes will produce a systematic change in the visibility only if hundreds of fringes are observed. The results show that each mode is spatially coherent within its divergence angle.

For a hologram with a size smaller than the coherence length, the fan-out (density of information) is diffractionlimited, and increasing the hologram size beyond the coherence length does not improve the fan-out due to the lack of coherence of the illuminating light. The coherence length thus determines $a_{0}$, the minimum (maximum) hologram size in which the density of information is coherence-limited 


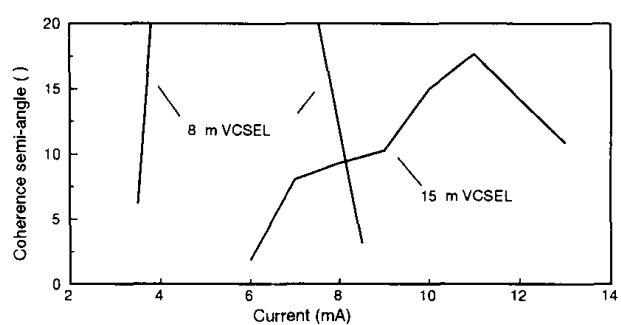

Fig. 3. Spatial coherence as a function of current for the $8-\mu \mathrm{m}$ and $15-\mu \mathrm{m}$ VCSEL's. For currents between 4 and $7.5 \mathrm{~mA}$ the coherence angle of the $8-\mu \mathrm{m}$ VCSEL is larger than what can be accurately measured with the system used. The FWHM emission angle of the lasers varies between 6 and 8 degrees.

(diffraction-limited). The worst case corresponds to multimode operation of the 8- $\mu \mathrm{m}$ VCSEL, for which we obtain $a_{0}$ $=0.6 \mathrm{~mm}$, while typical single-mode $a_{0}$ are in the $500-1000$ mm range. For pulsed operation, thermal chirp reduces $a_{0}$ of the single-mode to $16 \mathrm{~mm}$ for 5 -ns pulses and $1.5 \mathrm{~mm}$ for 50 -ns pulses. Therefore, from our experiments we conclude that neither the thermal chirp of short pulses, nor the spectral separation of modes are a limitation for interconnects employing holograms with dimensions $\leq 0.6 \mathrm{~mm}$, the density of information being diffraction-limited in that case by the size of the holographic element. The spatial coherence does not pose any limitation regardless of the hologram size for the VCSEL's studied, because the measured coherence angle is many times larger than the angle in which most of the energy is emitted.

To illustrate the above result, we used the VCSEL's to reconstruct a hologram recorded with a $\mathrm{He}-\mathrm{Ne}$ laser. In the recording process, the reference beam was set to have a divergence similar to that of the VCSEL, and the object beam was made to converge to a point $40 \mathrm{~cm}$ behind the holographic plate. Both beams overlapped on the plate and their directions formed an angle of 20 degrees. In this configuration the coherence requirements on the VCSEL are exaggerated with respect to the situation in the proposed interconnects due to the larger area $\left(\sim 10 \mathrm{~mm}^{2}\right.$ instead of $\left.0.2 \times 0.2 \mathrm{~mm}^{2}\right)$, larger distances, and the requirement of focusing, which could be performed using micro-lenses [2].

The resulting focal spot obtained in the reproduction of the hologram with the multi-mode $8-\mu \mathrm{m}$ VCSEL is shown in Fig. 4 for several currents. The focal spot is slightly broader at 4 and $8.5 \mathrm{~mA}$, where the output power is low and the bandwidth large. The optimum spot for this laser occurs at $5.5 \mathrm{~mA}$ where it emits its maximum power in a single mode. The FWHM of the spot in the focal plane in that condition was measured to be around $125 \mu \mathrm{m}$, which corresponds with the expected geometric magnification of the $8-\mu \mathrm{m}$ source. For $7 \mathrm{~mA}$ the multi-mode emission is resolved as a double spot in the focal plane. The thermal broadening of the spectrum of 5-ns laser pulses is too small to be resolved by the hologram. For shorter pulses, the thermal chirp would be even smaller and consequently would not impose limitations for the reconstruction of holograms for optical

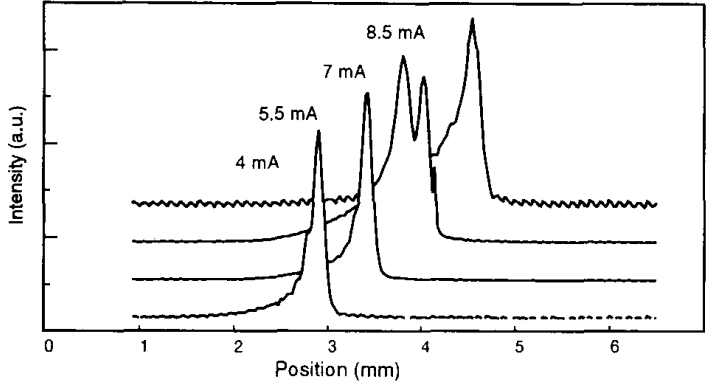

Fig. 4 Intensity profile of the $8-\mu \mathrm{m}$ VCSEL output in the focal plane of the hologram for different values of injected current.

interconnects. In contrast, for pulses longer than $50 \mathrm{~ns}$ the broadening was readily observable. It should be noted that the change in emission wavelength with a change in current from 4 to $8.5 \mathrm{~mA}$ produces a focal spot shift of around $2 \mathrm{~mm}$ for the conditions of our experiment. This stresses that the main limitations of VCSEL's are associated with thermal problems. Decreasing their power dissipation (e.g., reducing the resistivity of the mirrors) would also improve their coherence.

In conclusion, it was found that neither the thermal chirp nor the spectral separation of modes of VCSEL's limit the fan-out beyond the diffraction limit imposed by the size of the holographic element for interconnects employing holograms with dimensions $\leq 0.6 \mathrm{~mm}$.

\section{ACKNOWLEDGMENT}

The authors wish to thank V. Morozov for critical reading of the manuscript.

\section{REFERENCES}

[1] H. J. Caulfield, "Parallel $\mathrm{N}^{4}$ weighted optical interconnections," Appl. Opt., vol. 26, pp. 4039-4040, 1987.

[2] R. Feuerstein, D. C. O'Brien, A. Fedor, M. C. Chang, and L. H. $\mathrm{Ji}$, "Free space holographically interconnected counter," in Int. Conf. Optical Computing, Edinburgh. Aug. 1994.

[3] K. Zürl, E. Gluch, B. Schafer, and J. Schneider, "Smart pixels with VCSEL's: Potential and demonstration system," in Int. Conf. Optical Computing, Edinburgh. Aug. 1994.

[4] C. J. Chang-Hasnain, J. P. Harbison. G. Hasnain, A. C. Von Lehmen, L. T. Florez, and N. G. Stoffel, "Dynamic, polarization and transverse mode characteristics of vertical cavity surface emitting lasers," IEEE J. Quantum Electron., vol. 27, pp. 1402-1409, 1991.

[5] G. Hasnain, K. Tai, L. Yang, Y. H. Wang, R. J. Fischer, J. D. Wynn, B. Weir. N. K. Dutta, and A. Y. Cho, "Performance of gain-guided surface emitting lasers with semiconductor distributed Bragg reflectors," IEEE J. Quantum Electron., vol. 27, pp. 1377-1385, 1991.

[6] A. Yariv, Optical Electronics. New York: CBS College Publishing, 1985.

[7] D. Kuksenkov, S. Feld, C. Wilmsen, H. Temkin, S. Swirhun, and R. Leibenguth, "The linewidth and $\alpha$-factor in AlGaAs/GaAs vertical cavity surface emitting lasers," Appl. Phys. Lett., vol. 66, pp. 277-279, 1995.

[8] R. S. Geels, S. W. Corzine, and L. A. Coldren, "InGaAs verticalcavity surface-emitting lasers," IEEE J. Quantum Electron., vol. 27, pp. 1359-1367, 1991.

[9] B. Möller, E. Zeeb, U. Fiedler, and K. J. Ebeling, "Linewidth enhancement factor of vertical-cavity surface-emitting laser diodes," IEEE Photon. Technol. Lett., vol. 6, pp. 921-923, 1994. 